\title{
Challenging the Limits of Diffraction for Imaging, Biosensing and Optical Manipulation
}

\author{
Reuven Gordon
}

Dept. of Elec. \& Comp. Engineering, University of Victoria, Victoria, BC, Canada, V8W3P6

Diffraction limits the interfacing of light to the nanoscale, be it for imaging, biosensing, optical manipulation, lithography etc. Here, I will show ways of challenging (and overcoming) three limits of optical diffraction: Abbe's diffraction limit, Bethe's weak aperture transmission and Rayleigh's weak scattering by small particles. Both experimental and theoretical results will be shown.

Abbe's diffraction limit is well-known in the imaging community. This limit arises because the optical "information" associated with subwavelength features is bound evanescently to the object itself. One way to overcome this limit is to use a near-field probe, which, it can be argued, is invasive. Another approach is to try to extend the range of the near-field to achieve a subwavelength imaging spot-size at a distance comparable to the wavelength. This approach has already been demonstrated in the microwave regime and I will discuss ways of achieving similar results in the visible [1].

Bethe's aperture theory states that for an isolated subwavelength aperture, the transmission drops as the fourth power of the aperture diameter, and so it becomes exceedingly weak for small apertures. This has implications, for example, for aperture near-field scanning optical microscopy. While extraordinary optical transmission has shown that this limit breaks down for an array of such holes, the theory, as formulated by Bethe, considered only a single hole, and it was intended for use in waveguide and cavity systems. Here I show that even a single hole in a waveguide can have $100 \%$ transmission under the right conditions. Recently, this approach has been used to get $100 \times$ brighter near-field optical probes [2]. Rayleigh scattering of subwavelength particles limits the optical forces that can be used to manipulate such particles. As such, conventional optical trapping only works for particles greater than $100 \mathrm{~nm}$ in size. To trap smaller particles, it is possible to use a technique called self-induced back-action (SIBA). With SIBA, trapping of dielectric particles as small as $50 \mathrm{~nm}$ has been achieved [3]. I believe that this method has exciting prospects for trapping even the smallest virus particles, and experiments towards that goal are on-going.

\section{References:}

(1) R. Gordon, "Proposal for superfocusing at visible wavelengths using radiationless interference of a plasmonic array," Physical Review Letters, 102, 207402 (2009).

(2) L. Neumann., Y. Pang, A. Houyou., M. L. Juan., R. Gordon, N. F. van Hulst, "Extraordinary Optical Transmission Brightens Near-Field Fiber Probe," Nano Letters ASAP (2010).

(3) M. L. Juan, R. Gordon, Y. Pang, F. Eftekhari, R. Quidant, "Self-induced back-action optical trapping of dielectric nanoparticles," Nature Physics, 5, 915-919 (2009). 